\title{
Efectos de los diferentes colutorios para el tratamiento de la halitosis oral
}

\author{
López Jornet P*, Henarejos Hernández JL**, Saura Pérez M***, Camacho Alonso F**
}

\section{RESUMEN}

Objetivo: La halitosis intraoral se define como olor desagradable u ofensivo, que procede de la cavidad oral. La conexión entre mal aliento y compuestos volátiles sulfurados( CVS) fue establecida por Tonzetich 1977. Los diversos productos utilizados para el tratamiento de la halitosis tienen distintos objetivos: enmascarar, antibacteriano, convertir las formas volátiles de los CVS en no volátiles o la suma de los estos dos últimos. El objetivo es comparar la eficacia de los distintos tratamientos. Material y método: Se realizo un estudio piloto doble ciego, controlado se llevo a cabo en 40 sujetos voluntarios diagnosticados de halitosis intraoral repartidos distribuidos de forma aleatoria en 4 grupos de 10 sujetos, asignándole a cada grupo un tratamiento diferenteA (triclosan, fluorurosódico, cloruro de zinc, alcohol) B (triclosan, fluorurosódico, cloruro de zinc) fue C (lactato de zinc $0,14 \%$, chorhexidina $0,05 \%$, clorururo de cetilpiridino 0,05\%) y D con medicación placebo. El tratamiento lo realizo $10 \mathrm{ml} 2$ veces día durante 3 semanas. Los resultados se valoraron el nivel de CVS mediante Halimeter ${ }^{\circledR}(\mathrm{ppb})$. Resultados: No encontramos en este estudio diferencias estadísticas significativas ( $p=0,4932$ ) en la variable CVS medida mediante el halimeter ${ }^{\circledast}$. Los valores comparativos de $p$ en los 4 tratamientos ninguno fue menor de $p>0,01$. Conclusiones: Este protocolo no ha mostrado la eficacia de ninguno de los 4 tratamientos empleados en la disminución de VSC a corto plazo de la halitosis oral.

Palabras clave: halitosis, colutorio, compuestos volatiles sulfurados.

\begin{abstract}
Objective: The halitosis is defined as disagreeable or offensive odor, that comes from the oral cavity. The relationship between badly breath and volatile sulfur compounds (CVS) was established by Tonzetich 1977. The diverse products used for the treatment of the halitosis have different objectives: to mask, antibacterial, to turn the volatile forms of the nonvolatile CVS or the sum of the these two last ones. The objective is to compare the effectiveness of the different treatments. Material and method: Forty volunteers, participated in this study blind double pilot, controlled diagnosed of intraoral halitosis distributed of random form in 4 groups of 10 subjects, assigning to him to each group a treatment A (triclosan, zinc sodium fluoride, chloride, alcohol) B (triclosan, sodium fluoride, chloride of zinc) was C (zinc lactate $0.14 \%$, chlorhexidine $0.05 \%$, cetylpyridium chloride $0.05 \%$ ) and D with medication placebo. The treatment $10 \mathrm{ml} 2$ times day during 3 weeks. The results valued the level of CVS by means of Halimeter ${ }^{\text {(1) }}(\mathrm{ppb})$. Results: We did not find in this study significant statistical differences $(p=0,4932)$ in variable CVS measured by means of halimeter ${ }^{\text {i }}$. The comparative values of $p$ in the 4 treatments no was smaller of $p>0,01$. Conclusions: This protocol has not shown the effectiveness of any of the 4 treatments used in the diminution of short term VSC of the oral halitosis.
\end{abstract}

Key words: halitosis, mouthrinse, sulfur compounds.

* Prof. Titular Medicina Bucal. Universidad de Murcia.

** Licenciado Odontología. Universidad de Murcia.

*** Prof. Asociado Periodoncia. Universidad de Murcia.

López Jornet P, Henarejos Hernández JL, Saura Pérez M, Camacho Alonso F. Estudio piloto para valorar la eficacia de diferentes colutorios para el tratamiento de la halitosis oral. Av. Odontoestomatol 2003; 19-6: 275-282. 


\section{INTRODUCCIÓN}

La halitosis intraoral se define como olor desagradable u ofensivo, que procede de la cavidad oral. La conexión entre mal aliento y compuestos volátiles sulfurados( CVS) fue establecida por Tonzetich 1977 (1).

El 90\% de las causas de halitosis tienen su origen en la boca y el resto procede de puntos más distantes respiratorias, gastrointestinales, metabólicas, etc (26). La halitosis a nivel oral está provocada por la degradación bacteriana de partículas de alimentos, células, sangre y de algunos componentes de la saliva. Las proteínas y otros agentes químicos de estos materiales se van descomponiendo en componentes más simples como aminoácidos y péptidos. Durante el proceso se producen muchas sustancias volátiles (ácidos grasos y compuestos de sulfuro) (7).

Las situaciones locales orales que producen halitosis incluyen: gingivitis, periodontitis, caries extensas, falta de higiene oral y ulceraciones orales benignas o malignas (8-13). Bosy y colaboradores demostraron la primacía de la lengua como fuente de halitosis. Los individuos con revestimiento lingual tenían unas 25 veces mas bacterias por unidad de volumen de revestimiento que los sujetos sin el. La carga bacteriana total y la carga de bacterias anaerobias en la lengua pueden relacionarse con los resultados organolépticos de la lengua y boca (14-17).

Para valorar la halitosis se puede realizar mediante pruebas organolépticas, la intensidad del mal aliento observada por los jueces es normalmente graduada en tres o más niveles descriptivos (18). Otra alternativa para valorar este síntoma es utilizar cromatografía de gases, se demostró que el ácido sulfhídrico y el metil mercaptano son los constituyentes responsables de aproximadamente el $90 \%$ del olor; un tercer constituyente, en menor proporción, es el sulfuro de dimetilo. En la actualidad, la introducción de un monitor industrial (Halimeter ${ }^{\mathbb{E}}$ ) para la determinación de compuestos sulfurosos ha supuesto un importante avance. La correlación de los niveles encontrados con las pruebas organolépticas es buena (19-21).

En el tratamiento de la halitosis, la higiene oral y lingual es prioritaria, el efecto del cepillado sobre el dorso lingual es de corta duración, por lo que, el tratamiento puramente mecánico, no es suficiente para corregir el problema de la halitosis y debe ayudarse con un tratamiento antibacteriano químico, mediante la utilización de agentes antibacterianos vehiculizados en pastas, chicles o sobre todo en colutorios (22-25).

\section{Los agentes químicos utilizados son (26-36):}

- Cloruro de zinc. Produce interferencia en la producción y volatilización de los productos olorosos. El efecto del zinc es beneficioso a diferentes niveles: capacidad de formar compuestos no volátiles a partir de CVS; actividad antimicrobiana, ya que produce precipitación no selectiva de proteínas; reduce la degradación de elementos celulares en saliva la inhibición de la actividad de la tiolproteinasa; disminuye la permeabilidad de las membranas al paso CVS.

- Cloruro de cetilpiridinio y cloruro de bencetonio. Son compuestos derivados del amonio cuaternario con demostrada actividad antibacteriana in vitro. In vivo su actividad antibacteriana es más limitada debido a su rápido aclaramiento de la cavidad bucal.

- Compuestos fenólicos (timol, eugenol).Tienen no sólo actividad antibacteriana, sino también cierta actividad antinflamatoria. Dentro de este grupo destaca un compuesto químico, el triclosán. Es un antibacteriano potente que actúa a bajas concentraciones como bacteriostático. Ejerce su acción sobre la membrana citoplasmática, evitando la llegada a ella de aminoácidos esenciales. A mayores concentraciones, es bactericida, actúa desorganizando y destruyendo la membrana basal bacteriana. Aunque no posee la propiedad de sustantividad, su unión a otros compuestos como zinc facilita un aclaramiento más lento y, por lo tanto, una mayor eficacia in vivo.

Otro compuesto útil es la clorhexidina, a altas dosis se comporta como bactericida, alterando la permeabilidad de la membrana citoplasmática bacteriana. A bajas concentraciones es bacteriostática, interfiriendo el mecanismo de transporte fosfoenolpiruvato fosfotransferasa. Su actividad antimicrobiana es rápida y duradera disminuye la presencia de materia orgánica.

\section{MATERIAL Y MÉTODOS}

Estudio población. Este trabajo fue realizado en la 
clínica odontológica de la Universidad de Murcia, 40 pacientes fueron incluidos. Todos fueron voluntarios no remunerados. Se instruyó a los mismos sobre el objetivo del estudio. Además se les dieron instrucciones acerca de su preparación la noche anterior al estudio: evitar tomar cualquier tipo de alimentos que pudieran influir en el aliento, como el ajo, cebolla, especias fuertes, alcohol. Desde dos horas antes del estudio debían de abstenerse de: fumar, no tomar ningún alimento o bebidas excepto agua. No cepillarse los dientes o utilizar seda dental. No utilizar enjuagues o colutorios. Evitar el uso de perfumes o cosméticos.

Los criterios de inclusión para participar en el estudio, fue condición necesaria, ser mayor de edad, presentar halitosis clínica y haber firmado el consentimiento informado. Los criterios de exclusión: Fueron excluidos los individuos tratados con antibióticos un mes antes del estudio, pacientes que hubieran utilizado colutorios con clorhexidina u otro antimicrobiano el mes previo,pacientes con patologías respiratorias que utilizaran inhaladores, embarazadas y pacientes con ulceras bucales.

\section{Productos evaluados}

Se realizó un estudio piloto doble ciego, controlado se llevo a cabo en 40 sujetos voluntarios diagnosticados de halitosis intraoral repartidos y distribuidos de forma aleatoria en 4 grupos de 10 sujetos, asignándole a cada grupo un tratamiento diferente. Los productos fueron codificados por un agente externo a los investigadores, en botellas idénticas, y ni los sujetos evaluadores ni el investigador conocían los productos. Se estudiaron los siguientes productos:

A (triclosan+fluorurosódico+cloruro de zinc+alcohol).

B (triclosan+fluorurosódico+cloruro de zinc).

C (lactato de zinc $0,14 \%$, digluconato de clorhexidina 0,05\% + clorururo de cetilpiridino 0,05\%).

D con medicación placebo. (Con las mismas características y los mismos excipientes que el colutorio antihalitosis pero sin principios activos, alcohol o esencias).

Todos los participantes fueron instruidos de no usar productos de higiene bucal diferente al asignado y tampoco se permitio utilizar raspadores linguales. La pauta del tratamiento se realizo mediante la utilización de $10 \mathrm{ml}$ de colutorio dos veces día durante 3 semanas.

\section{Diseño experimental}

La duración del tratamiento fue de 3 semanas. En la tercera semana el paciente no utilizo colutorio solo pasta dentrifica. Se realizaron 4 visitas en las que se realizaron un total de 5 controles. Las variables principales fueron medición de los CSV y prueba organoléptica.

En la primera visita. Se realiza un control basal y se le asigna un colutorio previamente aleatorizado y el paciente se enjuaga y una hora después se realiza el control posterior. En la segunda visita se realiza una semana después de la primera en el que el paciente ha estado utilizando durante esa semana el colutorio asignado se realiza la medición organoléptica y medición de los CVS con el Halimeter ${ }^{\circledast}$.En la tercera visita a las dos semanas después de la primera se lleva a cabo de igual manera.

En la cuarta visita a las tres semanas de la primera el paciente durante esa semana no ha utilizado colutorio solamente pasta dental fluorada.

Para la determinación de los CSV, se utilizó el Halimeter $^{\circledast}$ modelo RH-17D (Fig. 1).

La medición con el Halimeter ${ }^{\text {( }}$ se efectúa de la siguiente forma:

1) Se calibra el aparato a cero en el medio ambiental.

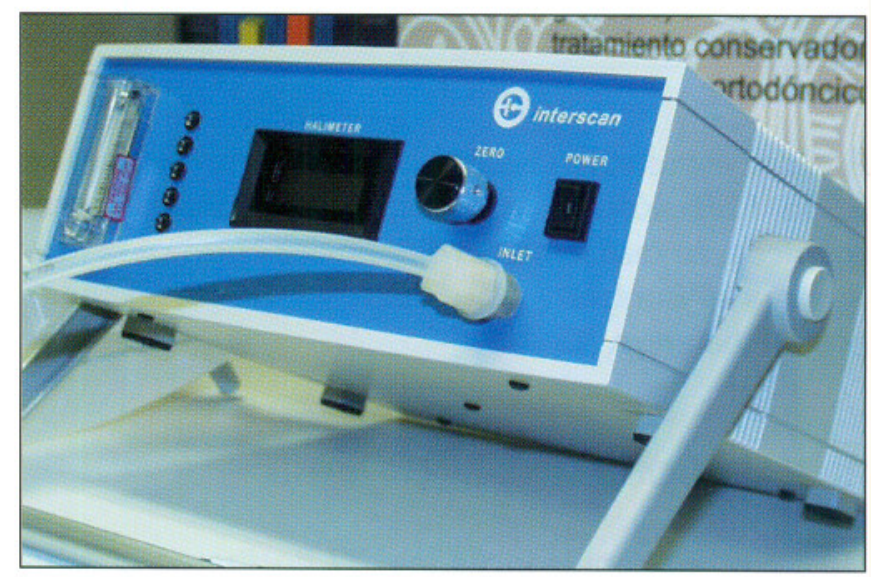

Fig. 1. Halimeter para medir CVS. 
2) Se introduce en la boca del paciente el extremo de la boquilla, aproximadamente $4 \mathrm{~cm}$. Durante toda la prueba la boca permanece entreabierta mientras que el paciente debe de respirar de forma relajada por la nariz. Es muy importante que no sople a través de la boquilla.

3) A los pocos segundos se anota la cifra más alta que aparece en la pantalla.

4) La boquilla debe ser sustituida en cada prueba, incluso para el mismo paciente.

5) Antes de hacer una nueva prueba se debe calibrar el aparato de nuevo a cero.

6) Se realizaron tres determinaciones consecutivas y se anotaron los tres picos más altos, al objeto de obtener un valor medio de dichos valores.

\section{Prueba organoléptica}

Se realizó inmediatamente después de haber medido con el Halimeter ${ }^{\circledast}$.El sujeto debe tener la boca cerrada durante un minuto, sin tragar, después de lo cual debe exhalar suavemente aire a una distancia de aproximadamente $10 \mathrm{~cm}$. de la nariz del clínico que va a juzgar el olor, quien inmediatamente debe valorarlo en una escala de 0 a 10 que se traslada a otra escala del 0 al 4 (Tabla1).

\begin{tabular}{|c|c|c|}
\hline \multicolumn{3}{|c|}{ TABLA 1.- VALORACIÓN ORGANOLÉPTICA } \\
\hline VALOR 0 - 10 & GRADO $0-4$ & TIPO DE HALITOSIS \\
\hline 0 & 0 & ausencia de halitosis \\
$1,2,3$ & 1 & halitosis leve \\
$4,5,6,7$ & 2 & halitosis moderada \\
8,9 & 3 & halitosis severa \\
10 & 4 & halitosis extrema \\
\hline
\end{tabular}

Análisis estadístico, se utilizo el paquete estadístico SPSS 11versión. Se utilizaron test estadísticos paramétricos, se realizaron las diferencias entre cada producto y el control y las diferencias de los distintos productos entre si.

\section{RESULTADOS}

De los 40 pacientes incluidos 37 finalizaron el estudio. La edad media fue de 33,70 años desviación típica 11,01 con un rango de edades de 21-55 años. El $48,6 \%$ eran varones y el $51,4 \%$ mujeres. De los sujetos estudiados 17 presentaban halitosis leve (45,9\%), 16 halitosis moderada $(43,2 \%)$ y $4(10,8 \%)$ halitosis severa medido siempre mediante escala organoléptica, en este estudio no había ningún caso de halitosis extrema. El valor medio de los CVS fue de $68,46 \mathrm{ppb}$. Se realizo un control a hora de haberse enjuagado con el colutorio asignado encontrando una disminución de los CVS de 49,92 ppb. Los valores medios de los CVS en la segunda visita fueron de $51,3 \mathrm{ppb}$, en la 3 visita de 55,7 ppb y en la cuarta visita en la cual el paciente no había utilizado colutorio únicamente dentífrico fluorado el valor de CVS fue de 58,5 ppb. Las características organolépticas referidas por el paciente el $89,2 \%$ encontraban un sabor agradable mientras que $10,8 \%$ no. También hubo un 3\% de los pacientes que encontraron sensación de ardor después de enjuagarse con el colutorio.

Al comprobar si existen diferencias estadísticamente significativas entre tratamientos en la variable CVS se ha hallado un valor de $\mathrm{p}=0,4932$ (Fg.2), consecuentemente no hallamos diferencias significativas entre los 4 tratamientos.

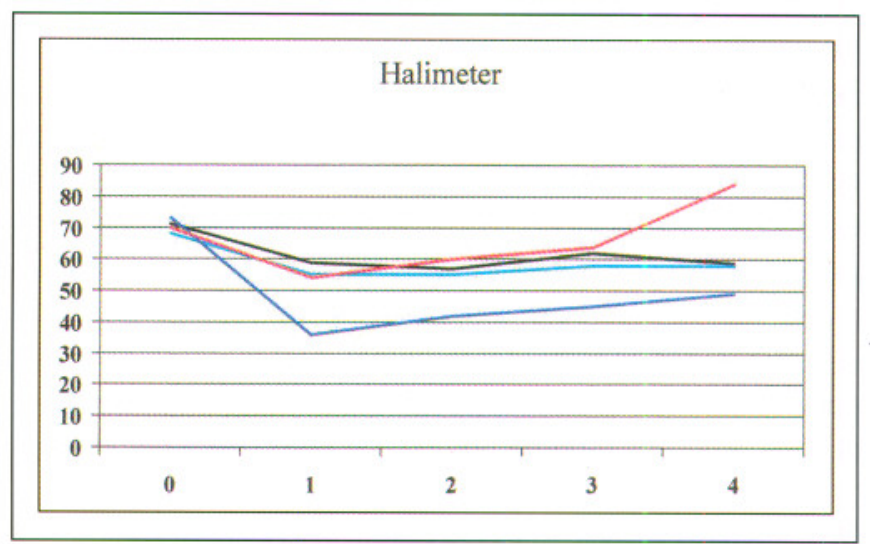

Fig. 2. Representación de los valores de los CVS medidos con el Halimeter ${ }^{8}$ durante el tratamiento con los distintos compuestos utilizados A ---, B -, C -, D -

Colutorio A versus colutorio $\mathrm{B} p=0,938$

Colutorio A versus colutorio $C p=0,5561$

Colutorio A versus placebo $\mathrm{p}=0,3420$

Colutorio B Versus colutorio C $p=0,609$

Colutorio $B$ versus placebo $p=0,058$

Colutorio $C$ versus placebo $p=0,1331$ 
En la variable exploración clínica no se observan diferencias significativas $p=0,2287$, si bien se encuentra que los colutorios $\mathrm{A}, \mathrm{B}, \mathrm{C}$ si que tienden a disminuir el mal aliento, estas diferencias no son significativas.

\section{DISCUSIÓN}

Este trabajo presenta los datos de un estudio clínico a corto plazo del tratamiento de diferentes colutorios Los agentes que parecen reducir el mal aliento tienen una actividad antibacterianana: clorhexidina, triclosan, cloruro de cetilpiridio, dióxido de cloro e iones de zinc. El empleo de las sales de zinc se basa en la fuerte afinidad de los iones zinc por los grupos tiol presentes en los compuestos volátiles (37).

Quirynen M et al (14) demostraron en estudio doble ciego realizado en voluntarios que el Listerine ${ }^{\circledR}$ reducía los niveles de bacterias odorigenas y las puntuaciones organolépticas reducía los compuestos volátiles sulfurados, este estudio fue uno de los primeros en comprobar que los agentes contra la halitosis eran efectivos si tenían un componente antimicrobiano.

Tonzetich (3) utiliza en 7 voluntarios sales de zinc y valora la eficacia de este colutorio a las 3 horas encontrando una disminución $80 \%$ en los CVS.

WinKel et al en 2003 (36) realizan un estudio doble ciego con un colutorio de lactato de zinc $0,14 \%$, clorhexidina $0,05 \%$, clorururo de cetilpiridino $0,05 \%$ y un placebo en 40 sujetos voluntarios sin enfermedad periodontal encontrando que es efectivo para el tratamiento de la halitosis reduciendo los componentes volatiles sulfurados de 292 a 172 ppb. Nuestros resultados indican que si bajan los niveles de CVS cuando se utiliza el colutorio pero estos no son significativos, quizas pueda deberse que el nivel de CVS es mas bajo que otros estudios como el trabajo realizado por Winket et al (36).

Respecto al tratamiento de la halitosis oral hay que recordar que se debe de realizar siempre el tratamiento de las enfermedades orales presentes como caries, gingivitis y periodontitis. También hay que valorar las prótesis desajustadas por lo que estos factores deben ser eliminados o subsanados. La xeros- tomía deberá ser tratada, cuando aparezca, mediante la utilización de técnicas de aumento del flujo. Otras medidas que son recomendables son las dietéticas como comer más a menudo, dejar espacios más cortos entre comidas, debe evitarse (ajo, cebollas) y se debe recomendar una dieta pobre en grasas y rica en frutas frescas y verduras(38-43).

Schmidt y Tarbet (43) realizaron estudio doble ciego en 62 sujetos utilizaron un colutorio de $\mathrm{ZnCl}$ los resultados indican una disminución significativa en la puntuación organoléptica y los compuestos volátiles sulfurados.

La forma de aplicación de estos productos tambien hay que considerarla en el trabajo realizado por Raven et al (27) encontraron que una combinación de citrato de zinc y triclosan administrados en un colutorio provoco una disminución del $66-83 \%$ de los compuestos volátiles sulfurados, mientras que la aplicación de estos dos componentes en un dentífrico produjo una reducción del $35 \%$.

La inclusión de alcohol en los colutorios es un tema controvertido, la presencia de alcohol en las formulaciones parece aumentar la efectividad del producto. Se ha discutido sobre el aumento de riesgo que supone la adición de alcohol y este debe evitarse en pacientes con mucositis, inmunocomprometidos, irradiados en cabeza y cuello y niños. Los datos de este estudio muestran que el $3 \%$ de todos los pacientes que utilizan colutorio tenian sensación de ardor. Para utilizar repetidamente un colutorio debe establecerse una seguridad. Noventa de 101 pacientes con que usaron colutorio con clorhexidina al $0,2 \%$ durante una semana respondieron a un cuestionario en relación con las reacciones adversas. El $88 \%$ de los pacientes refieriron al menos un síntoma, el 59\% experimento un cambio de sabor en la comida y el $25 \%$ una sensación urente en la punta de la lengua. Alrededor de $4 \%$ experimentaron sensación de adormecimiento de los tejidos. Debido a que la utilización fue unicamente de 1 semana cabe esperar mas problemas si el paciente lo utiliza durante periodos prolongados (13). También se ha utilizado como alternativa al tratamiento el peróxido de hidrógeno obteniéndose resultados contradictorios. El dióxido del cloro $\left(\mathrm{ClO}_{2}\right)$ al $0,1 \%$ se utiliza para el tratamiento de la halitosis por ser un agente que tiene una capaci- 
dad del redox alta para aquellos compuestos que contienen el azufre.

Kozlovsky et al (44), han utilizado para el control de la halitosis los colutorios en dos fases, se piensa que reducen el olor porque se produce una atracción polar entre las gotas de aceite y las células bacterianas. Otro trabajo realizado por Rosenberg emplean en individuos con resultados organolépticos inicialmente bajos, un colutorio de aceite agua con cloruro de cetilpiridio se mostró efectivo pero menos que la clorhexidina, en reducir la puntuación organoléptica (19).

Hay otras medidas generales, con el único objetivo de enmascarar, como son la utilización de colutorios de menta o clorofila (42). También se han publicado estudios utilizando agentes antimicrobianos tópicos, a dosis bajas, concretamente con clindamicina, en pacientes con cáncer para inhibir malodor oral (45). Frente a este panorama, los estudios clínicos son realmente necesarios para evaluar la eficacia de los distintos tratamientos que existen para el control de la halitosis oral.

\section{BIBLIOGRAFÍA}

1. Tonzetich J. Production and origin of oral malodor: A review of mechanisms and methods of analysis. J Periodontol 1977; 48: 13-20.

2. Tonzetich J. Oral malodor: An indicator of health status and oral cleanliness. Int.Dent J 1978; 28 : 309-19.

3. Tonzetich J,Ng SK Reduction of malodour by oral cleansing procedures Oral Surg Oral Med Oral Pathol Oral Radiol Oral End 1976; 42: 172-81.

4. Delanghe G, Ghyselen J, van Steenberghe D, Feenstra L. Multidisciplinary breath-odor clinic. Lancet 1997; 350: 187.

5. Kleinberg I, Westbay G. Oral malodor. Crit Rev Oral Biol Med 1990; 1: 247-59.

6. McDowell JD, Kassebaum DK. Diagnosing and treating halitosis. J Am Dent Assoc 1993; 124: 55-64.
7. Persson S, Edlund MB, Claesson R, Carlsson J. The formation of hydrogen sulfide and methyl mercaptan by oral bacteria. Oral Microbiol Immunol 1990; 5: 195-201.

8. Saura Pérez M, López Jornet P, Bermejo-Fenoll A. Diagnóstico y tratamiento de la Halitosis. RCOE 2001; 6, 2: 159-69.

9. Van Steenberghe D. Breath malodor. Curr Opin Periodontol. 1997; 4: 137-43.

10. Scully C, el-Maaytah M, Porter SR, Greenman J. Breath odor etiopathogenesis, assesment and management. Eur J Oral Sci 1997; 105: 287-93.

11. Yaegaki K., Coil JM. Clinical application of a questionnaire for diagnosis and treatment of halitosis. Quintessence Int 1999; 30: 302-6.

12. Coli J, Tonzetich J. Characterization of volatile sulfur compound production at individual gingival crevicular sites in humans. J Clin Dent 1992; 3: 97-103.

13. Bosy A, Kulkarni GV, Rosenberg M, McCulloch CAG. Relationship of oral malodor to periodontitis: Evidence of independence in discrete subpopulations. J Periodontol 1994; 65: 37-46.

14. Quirynen M, Mongardini C, van Steenberghe D. The effect of a one stage full-mouth disinfection on oral malodor and microbial colonization of the tongue in periodontitis patients. J Periodontol 1998; 69: 374-82.

15. Persson S, Claesson R, Carlsson J. The capacity of subgingival microbiotas to produce volatile sulfur compounds in human serum. Oral Microbiol Immunol 1989; 4: 169-72.

16. De Boever EH, Loesche WJ. Assessing the contribution of the anaerobic microflora of the tongue to oral malodor. J Am Dent Assoc 1995; 126: 1384-93.

17. De Boever EH, Loesche WJ. The tongue microbiota and tongue surface characteristics contribute to oral malodor. In: van Steenberghe D, 
Rosenberg M, eds. Bad Breath: A Multidisciplinary Approach. Leuven: Leuven University Press; 1996: 111-21.

18. Neiders M, Ramos B. Operation of bad breath clinics. Quintessence Int 1999; 30: 295-301.

19. Rosenberg M, McCulloch CAG. Measurement of oral malodor: Current methods and future prospects. J Periodontol 1992; 63: 776-82.

20. Rosenberg M, Kulkarni GV, Bosy A, McCulloch CAG. Reproducibility and sensitivity of oral malodor measurements with a portable sulphide monitor. J Dent Res 1991; 70: 1436-40.

21. Kostelc JG, Zelson PR, Preti G, Tonzetich J. Quantitative differences in volatiles from healthy mouths and mouths with periodontitis. Clin Chem 1981; 27: 842-5.

22. Bonesvoll P, Gjermo P. A comparison between chlorhexidine and some quaternary ammonium compounds with regard to retention, salivary concentration and plaque inhibiting effect in the human mouth after mouthrinses. Arch Oral Biol 1978; 23: 289-94.

23. Hoshi K, van Steenberghe D. The effect of tongue brushing or toothpaste application on oral malodor reduction. In: van Steenberghe $D$, Rosenberg M, eds. Bad Breath: A Multidisciplinary Approach. Leuven: Leuven University Press; 1996: 255-64.

24. Suárez FL, Furne JK, Springfield J,Levit MD Morning Breath odor: influence of treatements on sulfur gases. J. Dent Res. 2000; 10: 1773-7.

25. Reingewirtz Y, Girault O, Reingewirtz N, Senger B, Tenenbaum $\mathrm{H}$. Mechanical effects and volatile sulfur compound-reducing effects of chewing gums: Comparison between test and base gums and a control group. Quintessence Int 1999; 30: 319-23.

26. Moran J, Addy M, Kohut B, Hovliaras CA, Newcombe RG. Efficacy of mouthrinses in inhibiting the development of supragingival plaque over a 4-day period of no oral hygiene. J Periodontol 1994; 65: 904-7.

27. Raven SJ, Matheson JR, Huntington E, Tonzetich J. The efficacy of a combined zinc and triclosan system in the prevention of oral malodour. In: van Steenberghe D, Rosenberg M ed. Bad breath: a multidisciplinary approach. Leuven: Leuven Uni-verisity Press, 1996: 24154.

28. Moran J, Addy M, Jackson R, Newcombe RG. Comparative effects of quaternary ammonium mouthrinses on 4-day plaque regrowth. J Clin Periodontol 2000; 27: 37-40.

29. Renton-Harper P, Addy M, Moran J, Doherty FM, Newcombe RG. A comparison of chlorhexidine, cetylpyridinium chloride, triclosan and C31G mouthrinse products for plaque inhibition. J Periodontol 1996; 67: 486-9.

30. Roberts WR, Addy M. Comparison of the in vivo and in vitro antibacterial properties of antiseptic mouthrinses containing chlorhexidine, alexidine, cetylpyridinium chloride and hexetidine. Relevance to mode of action. J Clin Periodontol 1981; 8: 295-310.

31. Segreto VA, Collins EM, Beiswanger B, et al. A comparison of mouthwashes containing two concentrations of chlorhexidine. J Periodont Res 1986; 21: 23-32.

32. Addy M, Moran J, Wade WG, Jenkins S. The evaluation of toothpaste products in promoting gingival health. In: Embery G, Rölla G, eds. Clinical and Biological Aspects of Dentifrices. Oxford: United Press; 1992: 249-62.

33. Vandekerckhove BNA, van Steenberghe D, Tricio $\mathrm{J}$, Rosenberg D, Encarnacion M. Efficacy on supragingival plaque control of cetylpiridinium chloride in a slow-release dosage form. $\mathrm{J}$ Clin Periodontol 1995; 22: 824-9.

34. Rosenberg M, Gelernter I, Barki M, Bar-Ness R. Day-long reduction of oral malodor by a twophase oil: water mouthrinse as compared to 
chlorhexidine and placebo rinses. J Periodontol 1992;63:39-43

35. Roldan S, Winkel EG, Herrera D, Sanz M, Van Winkelhoff AJ. The effects of a new mouthrinse containing chlorhexidine, cetylpyridinium chloride and zinc lactate on the microflora of oral halitosis patients: a dual-centre, double-blind placebo-controlled study. J Clin Periodontol. 2003; 30: 427-34.

36. Winkel EG, Roldan S, Van Winkelhoff AJ, Herrera D, Sanz M.Clinical effects of a new mouthrinse containing chlorhexidine, cetylpyridinium chloride and zinc-lactate on oral halitosis. A dual-center, double-blind placebo-controlled study. J Clin Periodontol. 2003; 30: 300-6.

37. Addy M, Wade M. An approach to efficacy screening of mouthrinses: Studies on a group of French products.(I) Staining and antimicrobial properties in vitro. J Clin Periodontol 1995; 22: 718-22.

38. Umeda M, Contreras A, Chen C, Bakker I, Slots J. The utility of whole saliva to detect the oral presence of periodontopathic bacteria. J Periodontol 1998; 69: 828-33.

39. Willis CL, Gibson GR, Holt J, Allison C. Negative correlation between oral malodor and numbers and activities of sulphate-reducing bacteria in the human mouth. Arch Oral Biol 1999; 44: 665-70.

40. Yaegaki ,K. Sanada K Biochemical and Clinical Factors Influencing Oral Malodor in Periodontal Patients J. Periodontol.1992; 63: 783-9.
41. Yaegaki K, Sanada K. Volatile sulfur compounds in mouth air from clinically healthy subjects and patients with periodontal disease. J Periodont Res 1992; 27: 233-8.

42. Persson S. Hydrogen sulfide and methyl mercaptan in periodontal pockets. Oral Microbiol Immunol 1992; 7: 378-9.

43. Schmidt NF, Tarbet WJ. The effect of oral rinses on organoleptic mouth odor ratings and levels of volatile sulfur compounds. Oral Surg Oral Med Oral Pathol 1978; 45: 876-83.

44. Kozlovsky A, Goldberg S, Natour I, Rogatky-Gat A, Gelernter I, Rosenberg M. Efficacy of a 2phase oil:water mouthrinse in controlling oral malodor, gingivitis, and plaque. J Periodontol 1996; 67: 577-82.

45. Ogura T, Urade M, Matsuya T. Prevention of malodor from intraoral gauze with the topical use of clindamycin. Oral Surg Oral Med Oral Pathol 1992; 74: 58-62.

\section{CORRESPONDENCIA}

López Jornet P

Clínica Odontológica Universitaria

Medicina Bucal

Hospital Morales Meseguer

Adv Marques de los Velez s/n

Murcia 3008

Tel 968230061

Email: majornet@um.es 